\title{
Safety of Early Pharmacological Thromboprophylaxis after Subarachnoid Hemorrhage
}

\author{
Airton Leonardo de Oliveira Manoel, David Turkel-Parrella, Menno Germans, \\ Ekaterina Kouzmina, Priscila da Silva Almendra, Thomas Marotta, Julian Spears,
} Simon Abrahamson

\begin{abstract}
Objective: The recent guidelines on management of aneurysmal subarachnoid hemorrhage (aSAH) advise pharmacological thromboprophylaxis (PTP) after aneurysm obliteration. However, no study has addressed the safety of PTP in the aSAH population. Therefore, the aim of this study was to assess the safety of early PTP after aSAH. Methods: Retrospective cohort of aSAH patients admitted between January 2012 and June 2013 in a single high-volume aSAH center. Traumatic SAH and perimesencephalic hemorrhage patients were excluded. Patients were grouped according to PTP timing: early PTP group (PTP within 24 hours of aneurysm treatment), and delayed PTP group (PTP started $>24$ hours). Results: A total of 174 SAH patients (mean age $56.3 \pm 12.5$ years) were admitted during the study period. Thirty-nine patients $(22 \%)$ did not receive PTP, whereas 135 patients $(78 \%)$ received PTP after aneurysm treatment or negative angiography. Among the patients who received PTP, 65 (48\%) had an external ventricular drain. Twenty-eight patients (21\%) received early PTP, and 107 (79\%) received delayed PTP. No patient in the early treatment group and three patients in the delayed PTP group developed an intracerebral hemorrhagic complication. Two required neurosurgical intervention and one died. These three patients were on concomitant PTP and dual antiplatelet therapy. Conclusions: The initiation of PTP within 24 hours may be safe after the treatment of a ruptured aneurysm or in angiogram-negative SAH patients with diffuse aneurysmal hemorrhage pattern. We suggest caution with concomitant use of PTP and dual antiplatelet agents, because it possibly increases the risk for intracerebral hemorrhage.
\end{abstract}

RÉSUMÉ: Sécurité de la thromboprophylaxie pharmacologique précoce après une hémorragie sous-arachnoïdienne. Objectif: Les lignes directrices récentes sur la prise en charge de l'hémorragie sous-arachnoïdienne anévrismale (HSAa) recommandent la thromboprophylaxie pharmacologique (TPP) après l'oblitération de l'anévrisme. Cependant, aucune étude n'a examiné la sécurité de la TPP chez des patients présentant une HSAa. Le but de cette étude était donc d'évaluer la sécurité de la TPP précoce après une HSAa. Méthode: Cette étude rétrospective porte sur une cohorte de patients atteints d'une HSAa, hospitalisés entre janvier 2012 et juin 2013 dans un seul centre hospitalier à haut volume de HSAa. Les patients atteints de HSA traumatique ou d'hémorragie périmésencéphalique ont été exclus de l'étude. Les patients ont été répartis en deux groupes, selon le moment où la TPP a été administrée, soit dans les premières 24 heures ou plus de 24 heures après l'événement. Résultats: Cent soixante-quatorze patients atteints d'une HSA, dont l'âge moyen était de 56,3 $\pm 12,5$ ans, ont été entrés dans l'étude. Trente-neuf patients (22\%) n'ont pas reçu de TPP et 135 patients (78\%) ont été traités par TPP après le traitement de l'anévrisme ou après une angiographie négative. Parmi les patients qui ont reçu la TPP, 65 (48\%) avaient un drain ventriculaire externe. Vingt-huit patients $(21 \%)$ ont reçu la TPP dans les premières 24 heures et 107 patients $(79 \%)$ l'ont reçu plus de 24 heures après l'événement. Aucun patient dans le groupe qui a été traité tôt et 3 patients parmi ceux qui ont été traités tardivement ont présenté une complication hémorragique intracérébrale. Deux ont dû subir une intervention neurochirurgicale et un patient est décédé. Ces 3 patients recevaient la TPP et une double thérapie antiplaquettaire. Conclusions: Le début de la TPP dans les premières 24 heures pourrait être plus sûr après le traitement d'un anévrisme rompu ou chez les patients atteints d'une HSA dont l'angiographie est négative et chez qui on observe un pattern d'hémorragie anévrismale diffus. Nous recommandons la prudence lors de l'administration concomitante de la TPP et de deux agents antiplaquettaires à cause de la possibilité d'augmenter le risque d'hémorragie intracérébrale.

Keywords: Intracranial aneurysm, subarachnoid hemorrhage, venous thromboembolism, unfractionated heparin, low molecular-weight heparin, antiplatelet therapy

doi:10.1017/cjn.2014.16

Can J Neurol Sci. 2014; 41: 554-561

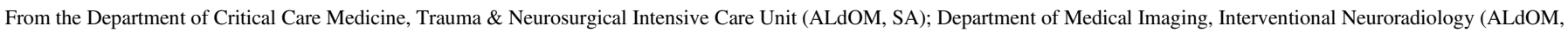

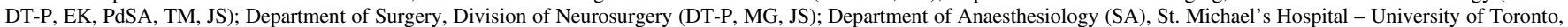
Toronto, Ontario, Canada.

Received February 14, 2014. Final Revisions Submitted April 25, 2014.

Correspondence to: Airton Leonardo de Oliveira Manoel, St. Michael's Hospital - University of Toronto, 30 Bond Street, Toronto, ON, Canada M5B 1W8. E-mail: airtonleo. manoel@gmail.com 


\section{BACKGROUND}

Venous thromboembolism (VTE) encompasses both deep vein thrombosis (DVT) and pulmonary embolism (PE), and it is a common complication in critically ill patients, including patients suffering from ischemic and hemorrhagic stroke. ${ }^{1,2}$ It represents a major health concern for hospitalized patients, with 180,000 in-hospital deaths per year in the United States from acute PE. ${ }^{3}$

The incidence of VTE in patients suffering from aneurysmal subarachnoid hemorrhage (aSAH) is reported to be in between $1.5 \%$ and $18 \% .^{4-6}$ Risk factors for VTE in the aSAH population include worse clinical status at presentation, ${ }^{7}$ prolonged length of hospital stay, ${ }^{5}$ and blood transfusion. ${ }^{8}$ Several strategies can reduce the risk of this complication, such as mechanical thromboprophylaxis (intermittent pneumatic compressions [IPC]) and pharmacological thromboprophylaxis [PTP] with low molecular-weight heparin [LMWH] or unfractionated heparin [UFH]).

Recently published guidelines recommend the use of IPC in all aSAH patients before aneurysm treatment. ${ }^{9,10}$ Although PTP should be withheld until the aneurysm is secured, it could be started immediately after endovascular treatment or at least after 12-24 hours after surgical clipping. ${ }^{9}$

Although the efficacy and safety of VTE prophylaxis has been established in other populations, no studies have specifically addressed the safety of PTP in aSAH patients. ${ }^{11}$ Therefore, the goal of this study was to evaluate the safety of PTP in aSAH patients.

\section{MethodS}

The clinical and imaging data of patients admitted with the diagnosis of SAH in a single high-volume SAH center (St. Michael's Hospital, Toronto) between January 2012 and June 2013 were retrospectively reviewed. In January 2013, an institutional SAH protocol was implemented that recommended IPC as soon as possible and at least before the treatment of a ruptured intracranial aneurysm. Pharmacological prophylaxis, consisting of UFH at a dose of 5,000 IU (subcutaneous [SC] every 12 hours) or dalteparin (5000 IU SC daily) was recommended within 24 hours of aneurysm treatment (regardless treatment modality) or after negative digital subtraction angiography (DSA). Mechanical prophylaxis is discontinued after the start of PTP. UFH and LMWH doses were adjusted according to the patient's bodyweight. According to the same protocol, PTP should be withheld $12 \mathrm{~h}$ before and after intracranial procedures (e.g. extremity venous duplex [EVD] removal, ventricular peritoneal [VP] shunt insertion). During this period, IPC is resumed.

Patients with traumatic SAH, perimesencephalic hemorrhage, or SAH from non-aneurysmal causes were excluded. Patients with diffuse aneurysmal hemorrhage pattern and negative DSA were kept in the analyses.

The time between aneurysm treatment and PTP initiation was recorded and patients were divided into two groups: (1) PTP within 24 hours of aneurysm treatment, as suggested in the SAH protocol (early PTP group), and (2) PTP started later than 24 hours after aneurysm treatment (delayed PTP group). Patients who did not receive PTP in the course of their intensive care unit (ICU) stay were analyzed separately and the reasons for lack of PTP were assessed. If no reason was recorded, the lack of PTP was considered not to be in compliance with the protocol, unless the patient was transferred to another hospital within 48 hours of aneurysm treatment. These patients were not included in the final analysis because the aim of the study was to assess the safety of early PTP in the aSAH population.

Routine surveillance with lower EVD ultrasonography (LEVDU) was not performed because this practice is controversial and not completely established. ${ }^{12,13}$ If there was a clinical suspicion for VTE, confirmed by the attending intensivist, an LEVDU or computed tomography pulmonary angiography (CTPA) was performed. ${ }^{14}$

The development of thrombocytopenia between days 4 and 14 after PTP initiation (i.e. total platelet count below $150 \mathrm{E} 9 / \mathrm{L}$ and/or a decrease $\geq 50 \%$ of platelet count from patient's baseline) was considered as possible heparin-induced thrombocytopenia type II (HIT II) according to the recommendations of the American College of Chest Physician Guidelines. ${ }^{15}$ The occurrence of new intracranial hemorrhage while the patient was on PTP was considered an intracranial hemorrhagic complication (IHC). The study was approved by the Hospital Review Ethics Board.

\section{Statistical Analysis}

Two-tailed Student's t-test with Welch's correction for heteroscedasticity was used to compare the characteristics of early and delayed PTP groups (Table 1). The two-sample test for equality of proportions without continuity correction was used to compare the early and delayed PTP group in terms of: (1) proportion of patients who received UFH or LMWH; (2) proportion of patients who developed IHC; (3) proportion of patients who had HIT II; and (4) proportion of patients who underwent LEVDU and/or CTPA. Last, patients who underwent LEVDU and/or CTPA were analysed in terms of proportions of individuals who were found positive for DVT or PE, respectively. A p value less than 0.05 was considered significant. All statistical analyses were performed in the R (v2.13.1) statistical environment.

\section{Results \\ Patient Characteristics}

One hundred seventy-four patients with SAH (mean age $56 \pm 13$ years) were admitted during the study period (Table 1). Thirty-nine patients $(22 \%)$ did not receive PTP during their ICU stay and the main reasons were: death before aneurysm treatment $(n=16)$, presence of an unsecured ruptured aneurysm with delayed treatment (i.e. treatment $>2$ weeks of hemorrhage; $n=9$ ), early repatriation to the referring hospital (i.e. repatriation within 48 hours of aneurysm treatment; $n=8$ ) and two patients were completely mobile. No apparent explanation for the lack of PTP was found in four patients, although it was indicated.

The remaining 135 patients $(78 \%)$ received PTP after aneurysm treatment or negative DSA and were included in the analysis. Twenty-eight patients (21\%) were in the early PTP group and 107 (79\%) in the delayed PTP group. In the delayed PTP group, 28 patients received PTP within 48 hours of aneurysm treatment, 29 within 72 hours, and the remaining patients received PTP after 72 hours of securing the aneurysm.

Both early and delayed PTP groups were similar in terms of their baseline characteristics. Eight patients presented with a diffuse aneurysmal SAH pattern, but had a negative DSA and therefore did not undergo aneurysm treatment. In total, 201 aneurysms were found (154 ruptured and an additional 47 unruptured aneurysms). 


\section{Table 1: Baseline characteristics}

\begin{tabular}{|c|c|c|c|c|}
\hline & No PTP & PTP within 24 hours & PTP later than $>24$ hours & p value \\
\hline Total & 39 & $28(21)$ & 107 (79) & NA \\
\hline Age & $58.7 \pm 12.6$ & $58 \pm 14$ & $55 \pm 12$ & 0.28 \\
\hline Females & $26(67)$ & $19(68)$ & $70(65)$ & 0.98 \\
\hline
\end{tabular}

\begin{tabular}{l|c|c|c|c}
\hline Modified Fisher score & \multicolumn{2}{l|}{} \\
\hline $\mathbf{1}$ & $6(15)$ & $1(7)$ & 1.00 \\
\hline $\mathbf{2}$ & $3(8)$ & $13(4)$ & $13(13)$ & $39(38)$ \\
\hline $\mathbf{3}$ & $5(13)$ & $12(44)$ & 0.30 \\
\hline $\mathbf{4}$ & $18(46)$ & $42(41)$ & 0.57 \\
\hline
\end{tabular}

\begin{tabular}{l|c|c|c|c}
\hline WFNS & \multicolumn{1}{l|}{} \\
\hline $\mathbf{1}$ & $13(33)$ & $5(46)$ & $48(46)$ & 1.00 \\
\hline $\mathbf{2}$ & $3(8)$ & $14(14)$ & 0.77 \\
\hline $\mathbf{3}$ & $0(0)$ & $2(7)$ & $0(8)$ & $13(12)$ \\
\hline $\mathbf{4}$ & $4(10)$ & $7(25)$ & 0.73 \\
\hline $\mathbf{5}$ & $14(36)$ & $21(20)$ & 0.77 \\
\hline
\end{tabular}

\begin{tabular}{l|c|c|c|c}
\hline Culprit rupture aneurysm & $26(67)$ & $28(100)$ & 0.53 \\
\hline Extra unruptured aneurysm & $3(8)$ & $8(29)$ & 0.47 \\
\hline & & & $21(20)$ \\
\hline Hydrocephalus on admission & $18(46)$ & $12(43)$ & $105)$ & $64(60)$ \\
\hline EVD & $15(38)$ & $11(40)$ & $54(50)$ & $19(18)$ \\
\hline VP shunt on discharge from hospital & NA & $1(4)$ & 0.40 \\
\hline
\end{tabular}

\begin{tabular}{l|c|c|c}
\hline Treatment modality & $21(75)$ & 7.00 \\
\hline Endovascular & $11(28)$ & $7(25)$ & $82(77)$ \\
\hline Surgical clipping & $3(8)$ & $0(0)$ & $17(16)$ \\
\hline Diffuse SAH pattern with negative angiography & $10(25)$ & 0.39 \\
\hline
\end{tabular}

\begin{tabular}{l|c|c|r|r}
\hline Antiplatelet therapy & \multicolumn{3}{|c|}{0} \\
\hline Aspirin & $0(0)$ & $0(15)$ & $9(8)$ & $10(9)$ \\
\hline Aspirin + clopidogrel & $0(0)$ & $0(0)$ & 0.21 \\
\hline
\end{tabular}

Data are shown as $\mathrm{n}(\%)$ or mean \pm standard deviation. The $\mathrm{p}$ values represent the comparison between the two groups that received PTP. EVD $=$ extremity venous duplex; NA = not applicable; $\mathrm{PTP}=$ pharmacological thromboprophylaxis; SA = subarachnoid; SAH = subarachnoid hemorrhage; WFNS = World Federation of Neurosurgical Societies.

Among the 135 patients who received PTP, 72 (53\%) received UFH and 67 (47\%) received LMWH. Four patients were started on LMWH, which was switched to UFH in the course of their ICU stay. An EVD was present in 65 (48\%) patients. Twenty-three patients received concomitant antiplatelet therapy (aspirin \pm clopidogrel), which included ten patients who received dual antiplatelet therapy after the insertion of an intracranial stent or flow diversion device.

\section{Ultrasound Doppler and CTPA Use and VTE Occurrence}

Twenty-five patients required LEVDU for clinically suspected DVT, three patients in the early and 22 in the late group (Table 2).
Additionally, 11 patients underwent CTPA to rule out PE, three in the early and eight in the late group. No patient in the early PTP group was diagnosed with VTE, whereas four patients in the delayed PTP group presented with VTE, which included two cases of DVT and two cases of segmental PE.

\section{Safety}

Four patients in each group developed thrombocytopenia after start of PTP. No patient in the early PTP group and three patients in the delayed PTP group developed IHC, respectively. These three patients received UFH and dual antiplatelet therapy (Table 3, Figure 1). 
Table 2: Ultrasound Doppler and computed tomography pulmonary angiography use VTE occurrence

\begin{tabular}{l|c|c|c}
\hline & $\begin{array}{c}\text { PTP within } \\
\text { 24 hours }\end{array}$ & $\begin{array}{c}\text { PTP later than } \\
\text { > 24 hours }\end{array}$ & Total \\
\hline Ultrasound Doppler, n (\%) & $3(11)$ & $22(21)$ & $25(18)$ \\
\hline $\begin{array}{l}\text { Computed tomography pul- } \\
\text { monary angiography }\end{array}$ & $3(11)$ & $8(7)$ & $11(8)$ \\
\hline Positive deep vein thrombosis & 0 & $2(2)$ & $2(2)$ \\
\hline Pulmonary embolism & 0 & $2(2)$ & $2(2)$ \\
\hline
\end{tabular}

$\mathrm{PTP}=$ pharmacological thromboprophylaxis; $\mathrm{VTE}=$ venous thromboembolism.

Data are shown as $\mathrm{n}(\%)$.

One patient developed a small intraventricular hemorrhage (Figure 1a) associated with the insertion of a VP shunt. The hemorrhage was not clinically significant and the patient did not require additional intervention. A second patient developed an acute-on-chronic subdural hematoma after the placement of a VP shunt (Figure 1b), requiring burr-hole drainage of the hematoma. This patient was discharged to a rehabilitation facility.

One patient with IHC died. This patient developed a thrombus in the proximal left anterior cerebral artery during the endovascular procedure. An intracranial stent was used for revascularization, which required dual antiplatelet therapy. The patient subsequently developed a large infarct in the left anterior cerebral artery territory. In the following days, while on UFH and dual antiplatelet therapy (i.e. aspirin and clopidogrel), the patient developed hemorrhagic conversion of the ischemic stroke (Figure 1c). A decompressive craniectomy was performed (Figure 1d) in an attempt to decrease the refractory elevated intracranial pressure, but the patient did not improve. After discussion with the family, the goals of treatment were changed to comfort measures and patient expired soon thereafter.

\section{Discussion}

This retrospective cohort of aSAH patients receiving PTP shows that early initiation of PTP (within 24 hours) after aneurysm treatment or negative angiography may be safe. Second, the concomitant use of PTP and dual antiplatelet therapy might increase the risk of IHC in this population.

Table 3: Safety

\begin{tabular}{l|c|c|c}
\hline & $\begin{array}{c}\text { PTP within } \\
\mathbf{2 4} \text { hours }\end{array}$ & $\begin{array}{c}\text { PTP later than } \\
\text { > 24 hours }\end{array}$ & Total \\
\hline $\begin{array}{l}\text { Heparin-induced } \\
\text { thrombocytopenia }\end{array}$ & $4(14)$ & $4(5)$ & $8(6)$ \\
\hline $\begin{array}{l}\text { Intracerebral hemorrhagic } \\
\text { complication }\end{array}$ & $0(0)$ & $3(3)$ & $3(2)$ \\
\hline $\begin{array}{l}\text { Bleeding complication requir- } \\
\text { ing intervention }\end{array}$ & NA & $2(2)$ & $2(1)$ \\
\hline $\begin{array}{l}\text { Death from hemorrhagic } \\
\text { complication }\end{array}$ & NA & $1(1)$ & $1(1)$ \\
\hline
\end{tabular}

$\mathrm{NA}=$ not applicable.

Data are shown as $\mathrm{n}(\%)$.

\section{Compliance}

Despite the existence of evidenced-based guidelines, ${ }^{16}$ pulmonary embolism continues to be the most preventable cause of in-hospital death, and only a minority of patients undergoing major surgery receive appropriate and timely VTE prophylaxis. ${ }^{17}$

In a large retrospective study including almost 150,000 surgical patients in the United States (approximately 17,000 neurosurgical patients), Deitelzweig et $\mathrm{al}^{17}$ showed that only $53 \%$ received appropriated and timely PTP, reflecting the difficulties of knowledge translation into clinical practice.

Likewise, Amin et al addressed the appropriateness of dose, type, and duration of VTE prophylaxis in a surgical population in US hospitals. The subgroup of neurosurgical patients had the lowest compliance to current published guidelines, with only $12 \%$ of patients receiving appropriate PTP. ${ }^{18}$

Specific to the SAH population, a retrospective study of 522 neurosurgical patients admitted over 11 years to a single surgical ICU showed that only 213 (40.8\%) of them received PTP, whereas $309(59.2 \%)$ never did. ${ }^{19}$ The SAH population had the lowest percentage of PTP among studied group. Out of 197 SAH patients, 125 (63\%) never received PTP.

\section{Timing}

Although there is compelling evidence that VTE is timedependent and can develop rapidly, ${ }^{20}$ initiation of PTP is usually delayed in a neurosurgical population because of concerns with IHC. ${ }^{21,22}$ Several theoretical concerns could explain the lack of or delay in PTP initiation after intracranial hemorrhage. Examples include risk of hematoma expansion, aneurysm rebleeding, the presence of an EVD, or the need for unpredictable neurosurgical procedures (e.g. EVD insertion). However, these delays are based on physicians' idiosyncrasies rather than on real risk cumulated from medical literature. ${ }^{21,22}$

Although the ideal timing between surgery and start of PTP is not completely clear, VTE prophylaxis using enoxaparin within 12-24 hours after surgery in combination with compressive stockings appears to be safe and superior to compressive stockings alone in patients undergoing craniotomy for tumor resection. ${ }^{23}$

Specifically, after the treatment of a ruptured aneurysm, if the aneurysm is completely excluded from the cerebral circulation, the immediate risk of aneurysm rebleeding is minimal. Additionally, endovascular coiling commonly uses systemic heparinization during the procedure with high doses of intravenous UFH to prevent thromboembolic complications. ${ }^{24}$ Therefore, the use of postoperative prophylactic dose of UFH or LMWH within 24 hours of endovascular treatment is probably of no consequence.

However, the initiation of anticoagulants is usually avoided or delayed after surgical treatment. Dickinson et $\mathrm{al}^{25}$ randomized patients undergoing craniotomies for treatment of brain tumors to receive IPC, enoxaparin, or enoxaparin plus IPC. The study was prematurely interrupted because of higher rates of IHC in the enoxaparin group compared with patients treated only with IPC (11\% vs. $0 \%$, respectively). This result is regularly cited and caused hesitancy in many neurosurgeons to start PTP after craniotomies.

Multiple studies have been published since then, ${ }^{26,27}$ including a meta-analysis of four randomized control trials ${ }^{28}$ showing no increased risk of postoperative bleeding after craniotomy with 


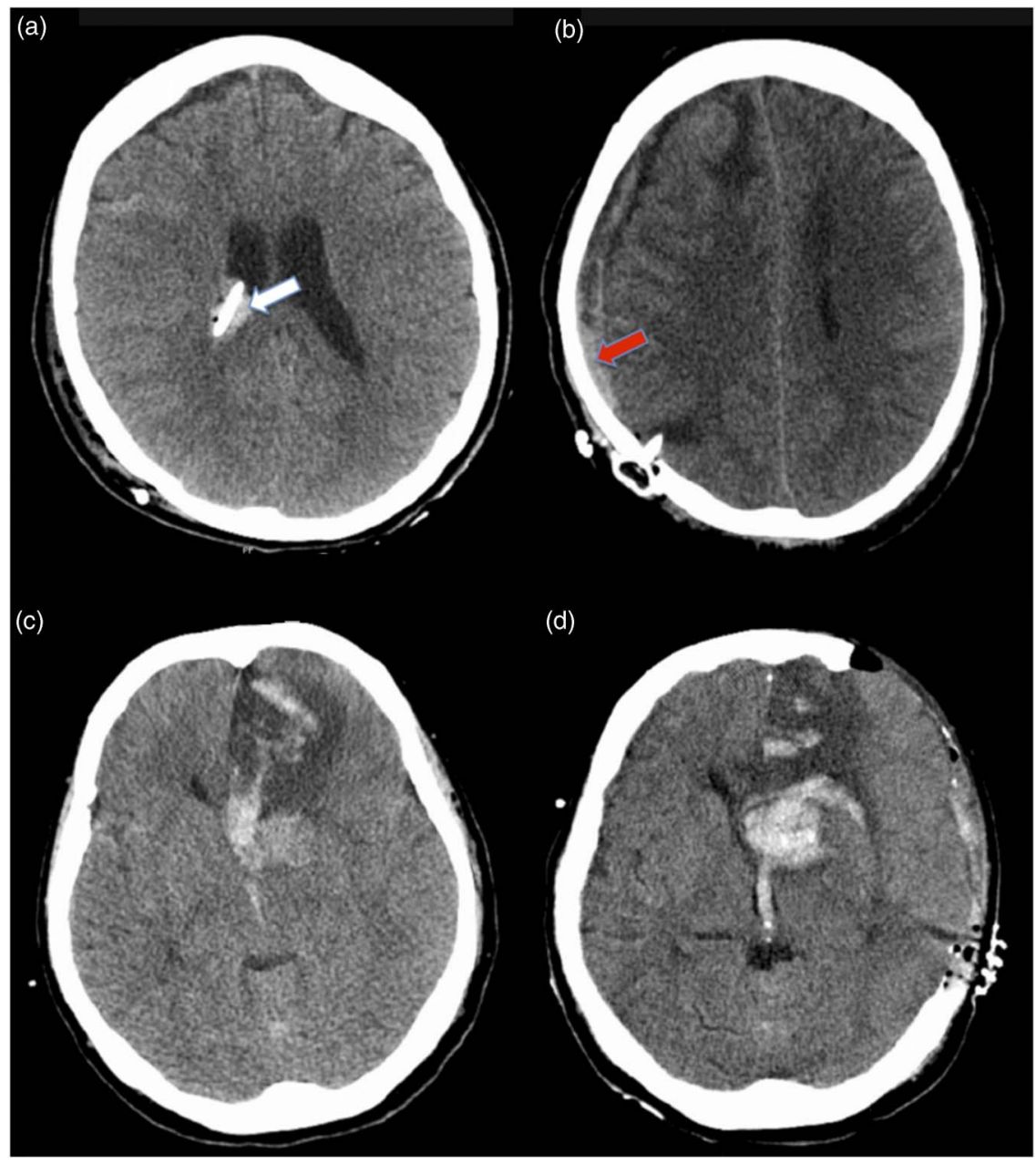

Figure 1: Intracranial hemorrhagic complication. (a) Right VP shunt inserted in the right lateral ventricle, with small amounts of blood along the tract and the ventricle (light arrow). (b) Mixed-density subdural hematoma along the right cerebral convexity (dark arrow). (c) Hemorrhagic transformation of a left anterior cerebral artery territory infarct. (d) After decompressive craniectomy.

heparin compared with control (i.e. either placebo or mechanical prophylaxis), with the number needed to treat to prevent a VTE of 7.7, and the number needed to cause a major bleeding of 102 , which highlights the benefits over the risks of PTP after craniotomy. Therefore, based on current available evidence, the practice of neurosurgeons to delay or avoid PTP after craniotomies might be unjustified.

The presence of an EVD has also been used as a relative contraindication to early PTP initiation. However, Tanweer et $\mathrm{al}^{29}$ have shown in a recent retrospective study including $34 \mathrm{SAH}$ patients, that the early start of PTP in patients with EVD was not associated with an increased rate of IHC.

In our cohort, 24 patients received PTP after craniotomy for aneurysm clipping, and none developed IHC. Seven of these patients received PTP within 24 hours of surgery. Although small in number, these seven clipped patients represent $25 \%$ of the early PTP group. Likewise, 65 patients received PTP in the presence of an EVD, with no IHC. No statistical difference in percentages of patients requiring EVD was found between the two groups to justify the delayed in PTP initiation.

\section{Ultrasound Doppler and CTPA Use and VTE Occurrence}

Although indications for tests to rule out VTE may vary among practitioners or institutions, clinical decision rules may not be as good as traditional physician judgment. Specifically, regarding VTE, it has been shown that unstructured clinician assessment performs better than clinical decision rules to estimate pretest probability for pulmonary embolism. ${ }^{14}$

Additionally, our study was not designed to assess efficacy, and the rate of symptomatic VTE was small; however, the incidence found was similar to other cohorts of $\mathrm{SAH}^{5}{ }^{5}$ patients undergoing cranial surgery, ${ }^{30}$ and immobile patients on PTP after ischemic stroke. $^{31,32}$ In our study, a number of asymptomatic DVTs might have been missed because LEVDU was not routinely performed. The routine use of LEVDU in the neurosurgical population has been shown to increase the detection of asymptomatic DVT and has been advocated by some, ${ }^{6,30,33}$ especially in poor-grade SAH patients. ${ }^{7}$ However, the clinical relevance of asymptomatic DVT is questionable and not completely understood. Furthermore the most recent American College of Chest 
Physicians Evidence-Based Clinical Practice Guidelines suggest against routine ultrasound screening for DVT. ${ }^{11}$

The low incidence of VTE could be also explained by the use of mechanical VTE prophylaxis in the form of intermittent pneumatic compression in all patients before the start of PTP. IPC has been shown to be effective to prevent VTE compared with PTP in neurosurgical patients. ${ }^{34}$ Additionally, in a large multicenter randomized trial, IPC have been shown to be effective in preventing DVT after ischemic stroke, and possibly improving survival. $^{35}$ In our SAH cohort, all symptomatic VTE complications developed in the delayed group. The use of IPC as a single modality of VTE prophylaxis in the SAH setting remains unclear and deserves additional research.

\section{UFH versus LMWH}

Our patient cohort had a similar incidence of thrombocytopenia compared with previous reports in the SAH population. ${ }^{36,37}$ The significantly lower risk for HIT when using LMWH has been extensively reported in the literature with different types of LMWH, including enoxaparin ${ }^{38}$ and dalteparin. ${ }^{39}$ Additionally, high doses of intravenous UFH are used during angiographic procedures, and it has been shown in the SAH population that HIT II is associated with the number of DSAs and not with the use of low-dose UFH for thromboprophylaxis. ${ }^{36}$

Although the use of enoxaparin appears safe, with no increased risk of intracranial hemorrhage after craniotomy, ${ }^{23}$ there are still concerns regarding the safety of PTP in the neurosurgical field. $^{21,22}$ The risk of intracranial hemorrhage has been thought to be associated with the type of agent rather than the start of PTP. ${ }^{5}$ In a randomized, double-blind, placebo-controlled trial, $170 \mathrm{aSAH}$ patients were randomized to enoxaparin (40 mg SC once daily) versus placebo for prevention of delayed cerebral ischemia, starting within 24 hours of ruptured aneurysm occlusion. ${ }^{40}$ Patients assigned to the enoxaparin group developed more IHC, which led to the conclusion that LMWH should be avoided in the early postoperative phase.

However, in another randomized trial comparing UFH and LMWH, Macdonald et al enrolled 100 patients to receive at induction of anaesthesia UFH 5000 IU SC and continued every 12 hours plus IPC versus dalteparin 2500 IU SC daily plus IPC. ${ }^{41}$ The majority of enrolled patients underwent craniotomy for tumor resection; however, approximately $15 \%$ in each group consisted of clipped ruptured cerebral aneurysms. No differences in terms of intraoperative blood loss, transfusion, or thrombocytopenia were found. The rates of VTE and hemorrhagic complications were not statistically different between the two groups. Our results do support the findings by Macdonald et $\mathrm{al}^{41}$ because none of the patients who received prophylactic treatment with $\mathrm{LMWH}$ developed IHC, even those who received it within 24 hours of aneurysm occlusion.

However, the use of UFH could be more convenient in the SAH population because of the unpredictable surgical procedures (e.g. EVD insertion), which requires withholding of PTP. The half-life of UFH is shorter and its effect can be completely reversed by protamine, making it more attractive for PTP in the SAH population.

\section{Antiplatelet Therapy Use in the SAH Population}

Twenty-three patients in this cohort received concomitant antiplatelet therapy and PTP, which includes ten patients that received dual antiplatelet therapy (aspirin and clopidogrel), because of the presence of an intracranial stent/flow diversion device implanted to treat the ruptured aneurysm. The aim of dual antiplatelet therapy in this setting is to prevent the catastrophic consequences of in-stent thrombosis.

In the acute phase of management of $\mathrm{SAH}$, recent data have shown that dual antiplatelet therapy may increase the risk for radiographic and symptomatic IHC. ${ }^{42,43}$ In a retrospective cohort of aSAH treated by endovascular coiling, ${ }^{43}$ the use of dual antiplatelet therapy after stent-assisted coiling was associated with significant higher rates of radiographic and symptomatic hemorrhage following EVD or VP shunt placement when compared with patients who underwent coiling without a stent, and therefore did not receive antiplatelet therapy ( $32 \%$ vs. $14.7 \%, \mathrm{p}=0.02$; and $8 \%$ vs. $0.9 \%, \mathrm{p}=0.03$, respectively).

Likewise, in another retrospective cohort of aSAH requiring VP shunt because of persistent hydrocephalus, ${ }^{42} 37$ of 206 patients underwent VP shunt placement. Interestingly, no new IHC occurred in patients that did not receive dual antiplatelet therapy; however, four patients presented with new IHC associated with VP shunt placement, and all of them were on dual antiplatelet therapy for stent-assisted coiling.

Three patients in our cohort developed a new IHC (one radiographic and two symptomatic hemorrhages). All of them were on concomitant PTP and dual antiplatelet therapy. This finding may again suggest that patients receiving dual antiplatelet therapy might have higher risk for new IHC. The use of only mechanical prophylaxis with IPC in this setting might be preferable; however, further research is required.

\section{Limitations}

Several limitations should be mentioned. First, the retrospective nature of our study prevents us correcting for confounders. Second, the small number of patients and the absence of patients receiving dual antiplatelet therapy in the early treatment group make the comparison between the two groups inappropriate. Last, the small number of surgically treated patients in this cohort could be seen as another possible limitation of the study; however, it actually represents a trend toward the endovascular approach in our institution and worldwide.

\section{Conclusion}

The use of early PTP may be safe after the treatment of a ruptured aneurysm or in angiogram-negative SAH patients with diffuse aneurysmal hemorrhage pattern. We suggest caution in the concomitant use of PTP and dual antiplatelet agents because it possibly increases the risk of intracerebral hemorrhagic complications.

\section{ACKNOWLEDGEMENTS AND FUNDING}

We thank Marlene dos Santos for her efforts in developing the research protocol and Research Ethic Board submission.

We acknowledge all the generous funding support for our clinical and research work provided by the Bitove Foundation. $\mathrm{TM}$ is the inventor and has patents for the Endovascular Clip System (eCLIPs).

\section{DisClosures}

Dr. Marotta is the inventor and has patents for the Endovascular Clip System (eCLIPs). 


\section{REFERENCES}

1. Gregory PC, Kuhlemeier KV. Prevalence of venous thromboembolism in acute hemorrhagic and thromboembolic stroke. Am J Phys Med Rehabil. 2003;82(5):364-9.

2. Skaf E, Stein PD, Beemath A, Sanchez J, Bustamante MA, Olson RE. Venous thromboembolism in patients with ischemic and hemorrhagic stroke. Am J Cardiol. 2005;96(12):1731-3.

3. Anderson FA, Zayaruzny M, Heit JA, Fidan D, Cohen AT. Estimated annual numbers of US acute-care hospital patients at risk for venous thromboembolism. Am J Hematol. 2007;82(9):777-82.

4. Serrone JC, Wash EM, Hartings JA, Andaluz N, Zuccarello M. Venous thromboembolism in subarachnoid hemorrhage. World Neurosurg. 2013;80(6):859-63.

5. Vespa P. Participants in the International Multi-Disciplinary Consensus Conference on the Critical Care Management of Subarachnoid Hemorrhage,. Deep venous thrombosis prophylaxis. Neurocrit Care. 2011;15(2):295-7.

6. Ray WZ, Strom RG, Blackburn SL, Ashley WW Jr, Sicard GA, Rich KM. Incidence of deep venous thrombosis after subarachnoid hemorrhage. J Neurosurg. 2009;110(5):1010-14.

7. Mack WJ, Ducruet AF, Hickman ZL, et al. Doppler ultrasonography screening of poor-grade subarachnoid hemorrhage patients increases the diagnosis of deep venous thrombosis. Neurol Res. 2008;30(9):889-92.

8. Kumar MA, Boland TA, Baiou M, et al. Red blood cell transfusion increases the risk of thrombotic events in patients with subarachnoid hemorrhage. Neurocrit Care. 2014;20(1):84-90.

9. Steiner T, Juvela S, Unterberg A, Jung C, Forsting M, Rinkel G. European stroke organization guidelines for the management of intracranial aneurysms and subarachnoid haemorrhage. Cerebrovasc Dis. 2013;35(2):93-112.

10. Diringer MN, Bleck TP, Claude Hemphill J, et al. Critical care management of patients following aneurysmal subarachnoid hemorrhage: recommendations from the neurocritical care society's multidisciplinary consensus conference. Neurocrit Care. 2011;15(2):211-40.

11. Guyatt GH. Executive summary. Antithrombotic Therapy and Prevention of Thrombosis, 9th ed: American College of Chest Physicians Evidence-Based Clinical Practice Guidelines. Chest. 2012;141(Suppl 2):7S-47S.

12. Schwarcz TH, Quick RC, Minion DJ, Kearney PA, Kwolek CJ, Endean ED. Enoxaparin treatment in high-risk trauma patients limits the utility of surveillance venous duplex scanning. J Vasc Surg. 2001;34(3):447-52.

13. Cipolle MD, Wojcik R, Seislove E, Wasser TE, Pasquale MD. The role of surveillance duplex scanning in preventing venous thromboembolism in trauma patients. J Trauma. 2002;52(3):453-62.

14. Penaloza A, Verschuren F, Meyer G, et al. Comparison of the unstructured clinician gestalt, the Wells score, and the revised Geneva score to estimate pretest probability for suspected pulmonary embolism. Ann Emerg Med. 2013;62(2):117-24.

15. Linkins LA1, Dans AL, Moores LK, et al. Treatment and Prevention of Heparin-Induced Thrombocytopenia: Antithrombotic Therapy and Prevention of Thrombosis, 9th ed: American College of Chest Physicians Evidence-Based Clinical Practice Guidelines. Chest. 2012;141(Suppl 2):e495S-530S.

16. Kakkar AK, Cohen AT, Tapson VF, et al. Venous thromboembolism risk and prophylaxis in the acute care hospital setting (ENDORSE Survey). Ann Surg. 2010;251(2):330-8.

17. Deitelzweig S, Lin J, Hussein M, Battleman D. Are surgical patients at risk of venous thromboembolism currently meeting the Surgical Care Improvement Project performance measure for appropriate and timely prophylaxis? J Thromb Thrombolysis. 2010;30(1):55-66.

18. Amin AN, Stemkowski S, Lin J, Yang G. Preventing venous thromboembolism in US hospitals: are surgical patients receiving appropriate prophylaxis? Thromb Haemost. 2008;99(4): 796-7.

19. Hacker RI, Ritter G, Nelson C, et al. Subcutaneous heparin does not increase postoperative complications in neurosurgical patients: an institutional experience. J Crit Care. 2012;27(3):250-4.

20. Chandra D, Parisini E, Mozaffarian D. Meta-analysis: travel and risk for venous thromboembolism. Ann Intern Med. 2009;151(3): 180-90.
21. Gnanalingham KK, Holland JP. Attitudes to the use of prophylaxis for thrombo-embolism in neurosurgical patients. J Clin Neurosci. 2003;10(4):467-9.

22. Carman TL, Kanner AA, Barnett GH, Deitcher SR. Prevention of thromboembolism after neurosurgery for brain and spinal tumors. South Med J. 2003;96(1):17-22.

23. Agnelli G, Piovella F, Buoncristiani P, et al. Enoxaparin plus compression stockings compared with compression stockings alone in the prevention of venous thromboembolism after elective neurosurgery. N Engl J Med. 1998;339(2):80-5.

24. Bendok BR, Hanel RA, Hopkins LN. Coil embolization of intracranial aneurysms. Neurosurgery. 2003;52(5):1125-30; discussion 1130.

25. Dickinson LD, Miller LD, Patel CP, Gupta SK. Enoxaparin increases the incidence of postoperative intracranial hemorrhage when initiated preoperatively for deep venous thrombosis prophylaxis in patients with brain tumors. Neurosurgery. 1998;43(5):1074-81.

26. Shlomi Constantini Andy Kanner, Friedman Adi, et al. Safety of perioperative minidose heparin in patients undergoing brain tumor surgery: a prospective, randomized, double-blind study. J Neurosurg. 2001;94:918-21.

27. Goldhaber SZ, Dunn K, Gerhard-Herman M, Park JK, Black PM. Low rate of venous thromboembolism after craniotomy for brain tumor using multimodality prophylaxis. Chest. 2002;122(6): 1933-7.

28. Iorio A, Agnelli G. Low-molecular-weight and unfractionated heparin for prevention of venous thromboembolism in neurosurgery: a meta-analysis. Arch Intern Med. 2000;160(15):2327-32.

29. Tanweer O, Boah A, Huang PP. Risks for hemorrhagic complications after placement of external ventricular drains with early chemical prophylaxis against venous thromboembolisms. J Neurosurg. 2013;119(5):1309-13.

30. Patel AP, Koltz MT, Sansur CA, Gulati M, Hamilton DK. An analysis of deep vein thrombosis in 1277 consecutive neurosurgical patients undergoing routine weekly ultrasonography. J Neurosurg. 2013;118(3):505-9.

31. Turpie AGG, Hull RD, Schellong SM, et al. Venous thromboembolism risk in ischemic stroke patients receiving extended-duration enoxaparin prophylaxis: results from the EXCLAIM study. Stroke. 2012;44(1):249-51.

32. Sherman DG, Albers GW, Bladin C, et al. The efficacy and safety of enoxaparin versus unfractionated heparin for the prevention of venous thromboembolism after acute ischaemic stroke (PREVAIL Study): an open-label randomised comparison. Lancet. 2007;369(9570):1347-55.

33. Henwood PC, Kennedy TM, Thomson L, et al. The incidence of deep vein thrombosis detected by routine surveillance ultrasound in neurosurgery patients receiving dual modality prophylaxis. J Thromb Thrombolysis. 2011;32(2):209-14.

34. Collen JF, Jackson JL, Shorr AF, Moores LK. Prevention of venous thromboembolism in neurosurgery: a metaanalysis. Chest. 2008; 134(2):237-49.

35. CLOTS (Clots in Legs Or sTockings after Stroke) Trials Collaboration, Dennis M, Sandercock P, Reid J, Graham C, Forbes J, Murray G. Effectiveness of intermittent pneumatic compression in reduction of risk of deep vein thrombosis in patients who have had a stroke (CLOTS 3): a multicentre randomised controlled trial. Lancet. 2013;382(9891):516-24.

36. Alaraj A, Wallace A, Mander N, Aletich V, Charbel FT, AminHanjani S. Risk factors for heparin-induced thrombocytopenia type ii in aneurysmal subarachnoid hemorrhage. Neurosurgery. 2011;69(5):1030-6.

37. Kim GH, Hahn DK, Kellner CP, et al. The incidence of heparininduced thrombocytopenia Type II in patients with subarachnoid hemorrhage treated with heparin versus enoxaparin. J Neurosurg. 2009;110(1):50-7.

38. Warkentin TE, Levine MN, Hirsh J, et al. Heparin-induced thrombocytopenia in patients treated with low-molecular-weight heparin or unfractionated heparin. N Engl J Med. 1995;332(20):1330-5.

39. PROTECT investigators for the Canadian critical care trials group and the Australian and New Zealand intensive care society clinical trials group, Cook D, Meade M, Guyatt G, et al. Dalteparin versus unfractionated heparin in critically ill patients. N Engl J Med. 2011;364(14):1305-14. 
40. Siironen J, Juvela S, Varis J, et al. No effect of enoxaparin on outcome of aneurysmal subarachnoid hemorrhage: a randomized, double-blind, placebo-controlled clinical trial. J Neurosurgery. 2003;99(6):953-9.

41. Macdonald RL, Amidei C, Baron J, et al. Randomized, pilot study of intermittent pneumatic compression devices plus dalteparin versus intermittent pneumatic compression devices plus heparin for prevention of venous thromboembolism in patients undergoing craniotomy. Surg Neurol. 2003;59(5):363-72; discussion 372-4.
42. Mahaney KB, Chalouhi N, Viljoen S, et al. Risk of hemorrhagic complication associated with ventriculoperitoneal shunt placement in aneurysmal subarachnoid hemorrhage patients on dual antiplatelet therapy. J Neurosurg. 2013;119(4):937-42.

43. Kung DK, Policeni BA, Capuano AW, et al. Risk of ventriculostomyrelated hemorrhage in patients with acutely ruptured aneurysms treated using stent-assisted coiling. J Neurosurg. 2011;114(4): 1021-7. 